\title{
Beware: The Misuse of Technology and the Law of Unintended Consequences
}

\author{
John M. Freeman \\ Emeritus Professor of Neurology and Pediatrics, Departments of Neurology and Pediatrics and the Berman Institute of \\ Bioethics, Johns Hopkins Medical Institutions, Baltimore, Maryland
}

\begin{abstract}
Summary: Electronic monitoring of the fetal heart rate during labor (EFM), originally designed to assess fetal stress and allow the early detection of the compromised fetus, has instead led to increasing maternal morbidity without decreasing fetal morbidity. The unintended consequences of this technologic advance have led to the creation of a pseudodisease and unwarranted intervention in response to its detection. Is it ethical to introduce a new technology without adequate assessment of its possible consequences? Are we about to repeat this (error resulting from the introduction of EFM?) There is increasing interest in monitoring the function of the newborn brain, to enable the early detection of subclinical seizures. The monitor may also be used for assessing brain function in older children and adults who are comatose or paralyzed and cannot appropriately respond to stimuli. Use of this amplitude-integrated
\end{abstract}

electroencephalography (aEEG) in the newborn for detection of seizures and other brain abnormalities is not dissimilar to the use of electronic fetal heart rate monitoring. Whether seizures or subclinical seizures themselves cause harm to the developing nervous system is unclear. The effectiveness of medications for treatment of seizures in the newborn has not been established. Therefore, the consequences of introducing automated EEG for the detection of subclinical neonatal seizures are likely to be similar to the results of the introduction of EFM: creation of another pseudodisease, followed by unwarranted intervention, and increased legal liability. What are the ethics of continued approval and introduction of unevaluated technology? What is the wisdom of its use? Beware of the unintended consequences. Key Words: Ethics of study design, fetal heart rate monitoring, aEEG, automated EEG.

\section{INTRODUCTION}

Patients want to be treated with the latest or best of whatever medication or technology is available. Physicians want to provide the most up-to-date treatment and diagnostic techniques. Information about what is supposedly the latest and best is often provided by colleagues on the golf course, at the latest dinner meeting sponsored by a company representative, or from an article in a throw-away journal. Sometimes information comes from patients who read about it in newspapers or magazines, or who saw it on television. Ideally, the "newest" or "best" comes from a refereed article in a recent medical journal. Only rarely have these latest therapies or technologies been evaluated properly in prospectively controlled studies, with the findings confirmed by replication, before widespread adoption. Only rarely are the possible consequences of their adoption foreseen. Is this

Address correspondence and reprint requests to: John M. Freeman, MD, 1026 Rolandvue Road, Ruxton, MD 21204. E-mail: jfreeman @jhmi.edu. rush-to-use by patients and physicians wise? Is the latest always the most effective? Is it appropriate? What are the ethics of the use of these inadequately tested technologies for experimental purposes in an individual? Is it ethical to use these inadequately tested technologies for what will be in effect experimental purposes in an individual?

Diseases are increasingly defined by technology, and new technology increasingly defines the "technologic invention of disease." ${ }^{1}$ Technology thereby establishes what constitutes disease. Blood pressure levels now define hypertension and often its treatment; blood sugar peaks and valleys define diabetes; and DNA is beginning to define multiple diseases. The electroencephalogram, once a tool used in the diagnosis and management of epilepsy, has begun to define epilepsy itself and is leading to treatment.

In a prescient article, Fisher and $\mathrm{Welch}^{2}$ note that more technology leads to more diagnoses and creates the potential for labeling and detection of what they term pseudodisease, a disease that might never become appar- 
ent to patients during their lifetime absent such testing. Diagnosis of these pseudodiseases leads to their respective treatments, and such treatments may lead to what they call tampering, a term the authors used to define "interventions to correct random, rather than systemic, variation." The ability to tamper can lead to lower treatment thresholds where the risks of the treatment outweigh its potential benefits.

Electronic fetal monitoring (EFM) is one example of a technology whose value is only now being challenged, 20 years after its introduction. Introduced with good intentions but inadequate assessment, EFM has become the standard of care in the delivery room. Its introduction, unfortunately, has led to the creation of a pseudodisease (supposed abnormalities of fetal heart rate), which in turn has led to interference with the delivery process on those grounds alone. The introduction of EFM appears to have caused more harm than good.

Amplitude-integrated EEG (aEEG) is a new technology being introduced into nurseries and intensive care units to detect subclinical seizures and to guide their treatment. ${ }^{3}$ aEEG is also being utilized in other situations to monitor brain function in patients who are comatose or paralyzed. Here we will confine our discussion to the neonate and the role of this new technology in the nursery. The introduction of aEEG into clinical use, without adequate testing and evaluation, may again produce unintended consequences: the creation of another pseudodisease leading to intervention with medications whose effects and effectiveness are unknown and possibly detrimental to the developing brain. The consequences may be similar to those already seen in the wake of EFM. Although the present discussion concerns primarily brain monitoring in the newborn, the concept applies to seizures at any age and to the development of new monitoring technologies for other diseases.

\section{ELECTRONIC FETAL MONITORING}

Since the end of the 19th century, the time of Little, Osler, and Freud, cerebral palsy (CP) has been known to be due to problems occurring with the fetus at birth. CP initially was attributed to brain damage from birth trauma, but as delivery techniques improved, $\mathrm{CP}$ was increasingly attributed to birth asphyxia. It is now known that only about $10 \%$ of $\mathrm{CP}$ is due to lack of oxygen. When intrauterine hypoxia or ischemia occurs from an event such as placenta previa or umbilical cord compression, the affected fetus may have bradycardia, and heart rate, respiration and motor function may be depressed at birth, manifesting signs termed neonatal encephalopathy. The newborn with such encephalopathy often goes (may go) on to have neurologic damage and cerebral palsy.

Abnormalities of fetal heart rate and rhythm have until recently been monitored by auscultation. In the late $1950 \mathrm{~s}$, it was hypothesized that if the decrease in fetal heart rate of such an infant could be detected sooner, before the hypoxic brain damage becomes severe, prompt delivery of the fetus might prevent or ameliorate the consequent brain damage and $\mathrm{CP} .^{4-6}$ Thus, the concept was born that EFM could be used to prevent cerebral palsy, and it was quickly adopted without prospective controlled studies of its efficacy or consequences. Although bradycardia and the encephalopathy detected are frequently ascribed to hypoxia, the signs and symptoms of the neonatal encephalopathy of asphyxia are mimicked by other, more prevalent, causes of CP.

Over recent decades, EFM increasingly has become the standard of care, and now $85 \%$ of deliveries are monitored electronically. ${ }^{6}$ Consequences of this monitoring are the subject of many recent reviews. ${ }^{7,8}$ To examine the consequences of electronic fetal monitoring, consider the following:

- Electronic fetal monitoring is easy to implement by nursing personnel, and less expensive than auscultation, but the data obtained are subjective, difficult to standardize, poorly reproducible, and subject to disagreement between observers. ${ }^{8}$

- In a recent study, only $0.2 \%$ of children with CP had manifested EFM abnormalities, and EFM had a $99.8 \%$ false positive rate. ${ }^{5}$

- Abnormalities such as bradycardia or late decelerations identified from EFM have led to a dramatic increase in the incidence of caesarean sections, with a consequent increase in maternal mortality and morbidity.

- EFM has produced no decrease in the incidence of CP.

- The increase use of EFM has coincided with, and has perhaps resulted in, the increase in what are called "bad baby" malpractice lawsuits.

EFM has thus led to the creation of a pseudodisease characterized by alterations in fetal heart rate and rhythm and often erroneously thought to be evidence of hypoxiaischemia.

In the hope of preventing permanent neurologic damage from this pseudodisease we now intervene ("tamper"), and this tampering has resulted in a dramatic increase in the rate of caesarean sections and an increase in maternal mortality and morbidity without having any effect on the incidence of cerebral palsy. There has been a concomitant increase in maternal hospitalization and in the cost of operative deliveries. ${ }^{9}$

Intervention, lack of intervention, and delay in intervention have also led to a marked increase in malpractice suits, with their own attendant costs of insurance and of defensive medicine. The plaintiffs' lawyers 
allege (erroneously) that if the physician had only recognized the abnormalities on the fetal heart rate monitor (i.e., the pseudodisease ) earlier and had intervened to deliver the baby (i.e., had "tampered") sooner, then the child would not have had CP.

The lessons from EFM offer a model and a cautionary tale for another device currently being introduced: aEEG. Beware!

\section{AMPLITUDE-INTEGRATED EEG}

Amplitude-integrated EEG is a currently developing technology used to record the EEG over long periods of time. The resultant compressed EEG can be used to monitor brain function and to detect baseline abnormalities, enabling intervention. Said to be predictive of future neurologic deficits by detecting abnormalities of background rhythms and neonatal seizures (and even subclinical seizures), it is as yet largely unproven and unstandardized and is potentially subject to many of the same unintended consequences seen with EFM. The physiologic basis and use of the aEEG are summarized by Rosen ${ }^{10}$ and Greisen. ${ }^{11}$

Brain dysfunction in the newborn is characterized by what is termed neonatal encephalopathy, demonstrated by apnea persisting 5-10 minutes after birth, bradycardia, acidemia, hypotonia, poor color, and neonatal seizures. Clinical seizures of early onset (within the first 12 hours of life) are often a marker of the severity of the encephalopathy and predictors of future dysfunction. Although this neonatal encephalopathy is often termed hypoxic-ischemic encephalopathy, the signs and symptoms of neonatal encephalopathy are not specific to hypoxic-ischemic brain injury and can more commonly result from maternal or fetal infection, genetic or metabolic problems, developmental abnormalities, bleeding, or stroke. Most neonatal seizures are of unclear cause, and only $6-10 \%$ of spastic CP is attributable to birth asphyxia. ${ }^{7}$ Infants with evidence of neonatal encephalopathy and seizures from whatever cause often (may) ultimately have $\mathrm{CP}$ and mental impairment.

Because clinical seizures in the newborn are associated with cerebral dysfunction, it has been hypothesized that the seizures themselves may either damage or exacerbate the damage of the neonatal brain. It is further hypothesized that if clinical seizures cause dysfunction, then subtle seizures, which are even more common and more difficult to detect reliably in the newborn, may also cause or exacerbate dysfunction. Further, it is thought that perhaps subclinical runs of spikes on the EEG (termed seizures by some) may, of themselves, ultimately cause dysfunction. However, although there is evidence that prolonged seizures in immature rodents may cause neuronal damage, there is no reliable evidence that subtle seizures in animals or humans either cause or exacerbate damage, and there is even less evidence that EEG abnormalities such as spikes on the human newborn EEG, now called subclinical electroencephalographically detected neonatal seizures, cause damage.

Neonatologists, eager to treat dysfunctional newborns, hope that early diagnosis and treatment of such seizures may allow development of interventions to decrease or prevent the subsequent neurologic impairment. It is unclear, however, whether any of these multiple causes will respond to currently available treatment or that their treatment will alter outcome. It is unclear that even clinically identifiable neonatal seizures are anything more than an indication of the severity of the then-current cortical dysfunction.

The standard EEG is the best method for confirming clinical seizures, and may also be the gold standard for detecting subclinical seizures. Emergent EEGs are difficult to obtain in the nursery, however. The electrodes are difficult to place and maintain properly; and the interpretation and identification of seizure events in the neonatal EEG require considerable training. Because subclinical seizures cannot reliably be detected without constant EEG monitoring, the use of automated EEG detection devices, which are more easily applied and thought to be easier to interpret, is advocated. The equipment is believed to permit detection of subclinical or subtle seizures as well as spikes on the neonatal EEG, even in the absence of clinical seizures. However, whether the spikes are indeed seizures, and whether or not they have any correlation with outcome, remains undetermined. There is even debate about whether the spikes seen on EEG may be harmful or beneficial. ${ }^{10,12-14}$

We do know that if the clinical EEG is normal or only mildly abnormal it reliably predicts a normal motor outcome for the infant, and that if the clinical EEG is severely abnormal or inactive it is ominous and highly predictive of death or severe motor disability. Intermediate forms are less reliably predictive. Few neonatal intensive care units have the equipment or expertise for prompt classical EEG recording and interpretation. Thus, there is some appeal to having the aEEG equipment available, despite report of poor reliability compared to and not supported by continuous classical EEG. ${ }^{15}$

If we could reliably detect subtle or subclinical seizures in this population, we would still have to ask the question, "Are the subtle neonatal seizures important, or are they merely evidence of a somewhat dysfunctional cortex?" At present we do not know if subtle seizures cause or contribute to neurologic dysfunction, and the importance of subclinical EEG-detected neonatal seizures is even less clear.

The advantage of aEEG is that it is easy to apply and, at least in experienced hands, may be useful in detecting abnormalities. When used to supplement the neurologic 
exam, aEEG may slightly improve the prediction of future disability. ${ }^{14}$ Whether, in routine nursery use, it will provide the same reliable detection of subtle seizures or of subclinical seizures as classical EEG, or will be subject to the same types of errors of interpretation seen with EFM is yet to be determined.

This leaves us with several problems. 1) Can we reliably detect subtle or subclinical seizures with aEEG? 2) If we detect them, are they important? 3) If they are important, can we treat them? 4) If we can treat them, will we prevent neurologic dysfunction?

At present, we do not know how to treat any neonatal seizures, whether clinical or subclinical. Although phenobarbital is the standard medication used, its effectiveness for this purpose has never been rigorously established. Writing for the Neurology Group on Neonatal Seizures, ${ }^{3}$ Clancy concludes, "There is a pressing need for randomized, placebo-controlled, ethically acceptable trials of phenobarbital efficacy and safety in the treatment of neonatal seizures."

The Neurology Group on Neonatal Seizures has designed a "multicenter, placebo-controlled, electroencephalographer-blinded study of phenobarbital versus placebo in a homogeneous group of newborns who are at high risk of developing early subclinical EEG-detected neonatal seizures." 3 The study plans to use video-EEG to detect subclinical seizures in infants (not newborns) at high risk for such seizures so that the effects of treatment with phenobarbital, a commonly used anticonvulsant in the treatment of neonatal seizures, can be evaluated. ${ }^{3}$

Again, this leaves us with questions:

- If phenobarbital is successful in totally eliminating these subclinical seizures, then what?

- Is this intervention beneficial in preventing the consequences caused by, or associated with, these subclinical seizures?

- If phenobarbital is unsuccessful in totally eliminating these subclinical seizures, then what?

- Were the subclinical seizures the cause of the dysfunction?

A more rational and persuasive study would first show that the subclinical EEG-detected seizures are associated with harm, and that the phenobarbital treatment prevents the harm due to the seizures detected. It would also have to prove that phenobarbital is not itself associated with harm. What are the ethics of using a technologically defined pseudodisease (such as subclinical spikes detected on aEEG) to evaluate the effectiveness and side effects of a treatment such as phenobarbital, of unknown efficacy for the prevention of a condition that may not exist?
- What are the ethics of introducing of new technologies such as EFM or aEEG to detect a pseudodisease of unknown consequence?

- Should such introduction be accompanied by assessment of its effectiveness and potential side effects?

a How should society deal with the creation of such pseudodiseases?

a How should we deal with the tampering type of interventions that result from this disease creation?

- How should society deal with a technology such as EFM which becomes established but then is found to have unintended consequences and to yield more complications than it prevents?

As Fisher and Welch ${ }^{2}$ have written, "Relatively little effort is devoted to evaluating current clinical practices, identifying their limitations, and advising clinicians about what not to do. Moreover, stakeholders in the increasingly market-driven U.S. health-care system have few incentives to explore the harms of the technologies from which they stand to profit."

EFM affords a cautionary tale of the unintended consequences of introducing technology without adequate evaluation, and without adequate consideration of its possible consequences. Without such preliminary safeguards, EFM did not meet what should be the ethical requirements for introducing new technologies. EFM is, however, a herald of other technologies to come.

What should we do now, 30 years after the introduction of EFM and in the face of its widespread use? Is there a way to reverse the tampering interference and the other consequences of our having created this pseudodisease?

- What should we do now, 30 years after the introduction of EFM and in the face of its widespread use? Is there a way to reverse the tampering interference and the other consequences of our having created this pseudodisease?

- Can we return to having nurses listen to the fetal heart rate, rather than watching the fetal heart rate monitor? (I strongly doubt it.)

- How can we teach physicians-and lawyers-the proper interpretation of the EFM recordings; the real meanings and significance of early and late decelerations; and the implications of tachycardia and bradycardia?

- What better ways can be found to identify the fetus suffering from hypoxia or ischemia and differentiate that infant from those with brain dysfunction due to genetic, developmental, or metabolic abnormalities or infections? 
- Does this early detection (of the child who will suffer dysfunction) even make a difference? Until we can find new approaches to mitigating the effect of each of these conditions and of treating the encephalopathy and the seizures, the reasons for early detection are unclear. (Perhaps hypothermia or one of the newer neuroprotective therapies may be successful.)

One thing we can do is to effect changes in the malpractice system-what has been called the malpractice lottery system ${ }^{16}$ - and thus escape from the current malpractice-sensitive, defensive approach to delivery, which is harmful to patients, infants, and physicians to the benefit of no one.

Neonatal seizures are frequently seen in association with neonatal encephalopathy, but that does not mean they cause the ensuing cerebral palsy. Abnormalities on the EEG without clinical seizures (i.e., subclinical electroencephalographically detected seizures) are not the same as clinical seizures, and their relationship to future neurologic dysfunction is purely speculative. Thus, use of aEEG to detect these abnormalities is bound to result in the creation of another new pseudodisease.

If the lessons of EFM remain unlearned, we face yet more questions.

- What will be the likely consequences of the current introduction of a EEG for detection of subclinical neonatal seizures?

- What will be the consequence of treatment, given that these subclinical seizures may be of only theoretical importance?

- To what extent is it ethical to continue to approve and introduce such untested technology?

Treatment of true clinical seizures in the newborn has never been subjected to appropriate controlled clinical testing. ${ }^{3}$ Creation of this new pseudodisease of subclinical electroencephalographically detected seizures will, like the similarly untested introduction of EFM, lead to tampering interventions (i.e., to new therapies treating a condition of uncertain and possibly no importance), with similar and as yet unknown unintended consequences.

Approval of aEEG machines for early detection of these subclinical seizures is thus in fact unethical, because it will lead to the creation and detection of a pseudodisease of unknown consequence and to its unproven treatment. Perhaps aEEG machines could be useful in research, to determine the importance of the neonatal spiking detected. If spiking leads to neurologic impairment, that would be good to know, and research might lead to new treatments for an actual condition.

Perhaps the FDA should approve new technology only for specific tested use, and hospitals should consider other uses to be off-label research, requiring appropriate IRB oversight. In our current state of ignorance of these factors, however, there is great pressure from companies and from researchers with potential conflicts of interest to introduce new and untested technology into clinical use. This likewise is, in my opinion, unethical.

\section{CONCLUSION}

The multiple challenges to conducting trials of neuroprotection in asphyxiated term newborns are indeed daunting, and are well described by Shankaran and Laptook. ${ }^{17}$ These challenges include everything from establishing a diagnosis of hypoxic-ischemic encephalopathy to patient selection, and from the therapeutic window for intervention to the safety and side effects of the intervention. Duration and follow-up variables likewise present challenges.

Preliminary studies in animals and neonates are documenting that hypothermia may be beneficial in preventing or ameliorating the effects of hypoxic-ischemic encephalopathy-if the hypothermic state is induced soon after the insult. ${ }^{18}$

In an editorial focusing on trials of medications, the Society for Clinical Trials ${ }^{19}$ emphasizes the need for care in trial design, the need to avoid duplicative research, the importance of ethical relevance to clinical practice, and the role of cost in the ethical calculus. Although the editorial does not discuss the role of these parameters in the introduction of new technology, they should be of equal concern. Others have focused on the need for costeffectiveness studies. ${ }^{20,21}$ Neither of those two reports directs attention to the unintended consequences of research and technology, nor does the FDA's white paper ${ }^{22}$ outlining the agency's plans for encouraging and facilitating new technology.

Until the FDA and the medical community are required to go beyond reviewing studies of the safety and efficacy of medications and technology and begin to address their consequences-both intended and unintended-and the cost-effectiveness of the new medications and new technology, the ethics of the approval process will remain questionable. As with other areas of newborn screening technology, we should indeed "proceed with caution." 23 Beware!

\section{REFERENCES}

1. Hofmann B. The technological invention of disease. J Med Ethics 2001;27:10-19.

2. Fisher ES, Welch HG. Avoiding the unintended consequences of growth in medical care: how might more be worse? JAMA 1999; 281:446-453.

3. Clancy RR. Summary proceedings from the Neurology Group on Neonatal Seizures. Pediatrics 2006;117:S23-S27.

4. Graham EM, Peterson SM, Christo DK, Fox HE. Intrapartum electronic fetal heart rate monitoring and the prevention of perinatal brain injury. Obstet Gynecol 2006;108:656-666.

5. Royal College of Obstetricians and Gynaecologists. The use of electronic fetal monitoring. The use and interpretation of cardio- 
tocography in intrapartum fetal surveillance. London: RCOG Press; 2001.

6. Alfirevic Z, Devane D, Gyte GM. Continuous cardiotocography (CTG) as a form of electronic fetal monitoring (EFM) for fetal assessment during labour. Cochrane Database Syst Rev 2006;3: CD006066.

7. Nelson K. Can we prevent cerebral palsy? N Engl J Med 2003; 349;18:1765-1769.

8. Greene MF. Obstetricians still await a deus ex machina. N Engl J Med 2006;355:2247-2248.

9. Freeman JM. The use of amplitude-integrated electroencephalography: beware of its unintended consequences. Pediatrics 2007; 119:615-617.

10. Rosen I. the physiological basis for continuous electroencephalogram monitoring in the neonate. Clin Perinatol 2006;33:593-611, v.

11. Greisen G. Brain monitoring in the neonate: the rationale. Clin Perinatol 2006;33:613-618, v-vi.

12. Rogawski MA. Point-counterpoint: do interictal spikes trigger seizures or protect against them? Epilepsy Curr 2006;6:197-198.

13. Staley KJ, Dudek FE. Interictal spikes and epileptogenesis. Epilepsy Curr 2006;6:199-202.

14. Avoli M, Biagini G, de Curtis M. Do interictal spikes sustain seizures and epileptogenesis? Epilepsy Curr 2006;6:203-207.

15. Murray DM, Ryan CA, Boylan GB, et al. Prediction of seizures in asphyxiated neonates: correlation with continuous videoelectroencephalographic monitoring. Pediatrics 2006;118:41-46.

16. Freeman AD, Freeman JM. No-fault cerebral palsy insurance: an alternative to the obstetrical malpractice lottery. J Health Polit Policy Law 1989;14:707-718.

17. Shankaran S, Laptook A. Challenge of conducting trials of neuroprotection in the asphyxiated term infant. Semin Perinatol 2003; 27:320-332.

18. Gunn AJ, Battin M, Gluckman PD, Gunn TR, Bennet L. Therapeutic hypothermia: from lab to NICU. J Perinat Med 2005;33: $340-346$

19. Goodman SN. Ethics and evidence in clinical trials. Clin Trials 2005;2:195-196.

20. Hlatky MA, Owens DK, Sanders GD. Cost-effectiveness as an outcome in randomized clinical trials. Clin Trials 2006;3:543-551.

21. Gray AM. Cost-effectiveness analysis alongside randomized clinical trials. Clin Trials 2006;3:538-542.

22. U.S. Food and Drug Administration. Innovation or stagnation? Challenge and opportunity on the critical pathway to new medical products. FDA, 2004 [Available at: http://www.fda.gov/oc/ initiatives/criticalpath/whitepaper.pdf].

23. Botkin JR, Clayton EW, Fost NC, et al. Newborn screening technology: proceed with caution. Pediatrics 2006;117:1793-1799. 